\title{
Descriptive/Inferential Cognitive Processes and Evaluative Cognitive Processes: Relationships Among Each Other and with Emotional Distress
}

\author{
Andreea Vîslă • Martin grosse Holtforth • \\ Daniel David
}

Published online: 26 February 2015

(C) Springer Science+Business Media New York 2015

\begin{abstract}
We aimed to delineate key constructs from two forms of cognitive-behavioral therapy: cognitive therapy and rational-emotive behavior therapy. Furthermore, we aimed to investigate the interrelations among each other and with emotional distress. The key constructs of the underlying theories of these therapies (i.e., descriptive/inferential beliefs, evaluative beliefs) are often treated together as distorted cognitions and included as such in various scales. We used a cross-sectional design. Seventy-four undergraduate students (mean age $=24.68$ ) completed measures of automatic thoughts and emotional distress. Three therapists trained in cognitivebehavioral therapy divided automatic thoughts into descriptive/inferential beliefs and evaluative beliefs by consensus. Correlation and mediation analyses were performed. These constructs showed medium to high associations to each other and to distress. The relationship between descriptive/inferential beliefs and distress was mediated by evaluative beliefs. Descriptive and inferential cognitions may not produce emotions without first being appraised in terms of personal relevance.
\end{abstract}

\footnotetext{
A. Vîslă $(\bowtie) \cdot$ D. David

Department of Clinical Psychology and Psychotherapy, Babeş-Bolyai University, No. 37, Republicii St., 400015 Cluj-Napoca, Romania

e-mail: andreea.visla@gmail.com
}

A. Vîslă · M. g. Holtforth

Department of Psychology, University of Zurich, Zurich, Switzerland

M. g. Holtforth

Department of Psychology, University of Bern, Bern, Switzerland

D. David

Department of Oncological Sciences, Icahn School of Medicine at Mount Sinai,

Box 1130, New York, NY, USA 
Keywords Cognitive-behavioral therapy - Descriptions/inferences - Evaluations · Emotional distress · Mediation

\section{Introduction}

In the development and maintenance of psychological distress, cognitive processes have been postulated as essential components since the early 1950s (Broadbent 1958; Chomsky 1959; Miller 1956). Cognitions are one central element in cognitive-behavioral therapy (CBT), one of the most extensively researched forms of psychotherapy. Although cognitive-behavioral therapies are among the best empirically supported approaches for a wide range of psychological problems, the efficacy of CBT is insufficient for some problems, suggesting that further improvements in CBT strategies and related theories are still needed (Hofmann et al. 2012). According to CBT theory, the impact of various activating life events (e.g., failure on an exam; A) on different psychological consequences (e.g., feelings and behavioral, cognitive, and psychophysiological reactions; C) is mediated by information processing (cognitions/beliefs; B). However, CBT approaches differ in the relative importance ascribed to certain cognition types in generating emotions.

Rational-emotive behavior therapy (REBT; Ellis 1994) and cognitive therapy (CT; Beck 1976 and its update in Beck 1995) differ in the relative emphasis placed on "hot" versus "cold" cognitions (David et al. 2004; David and Szentagotai 2006). Beck's CT focuses primarily on "cold" cognitions, that is, mental representations of relevant circumstances, which reflect knowledge or beliefs about what is happening (Lazarus 1991), in the forms of dysfunctional/distorted descriptions and inferences. The central difference between these two concepts is that descriptions merely present an observed fact (e.g., "It is a crowded auditorium"), whereas inferences extend further from the perceived fact (e.g., "They will laugh at me"). In contrast, REBT focuses mainly (but not exclusively) on "hot" cognitions, also called appraisals or evaluative cognitions (i.e., rational and irrational beliefs), which refer to how these representations are appraised with respect to their significance for personal well-being (Lazarus 1991) (e.g., "They must not laugh at me and if they do it is awful"; "demandingness and awfulizing" irrational beliefs). In a broader sense, REBT admits that descriptions and inferences are relevant to emotion because they are the data that the person evaluates with respect to their motivational relevance. However, without being appraised in terms of rational (e.g., "I would like them not to laugh at me, but I can accept that I am a worthy person even if they do laugh") or irrational beliefs (e.g., They must not laugh at me and if they do it is awful), they do not directly generate emotions (Ellis 1994). Therefore, REBT states that descriptions and inferences do relate to emotions, but not directly.

Although this distinction is a key one in the general psychology theories (for the distinction between appraisals vs. attributions, see Lazarus 1991), sometimes in CT this distinction is overlooked in the name of clinical relevance. Indeed, because at the phenomenological level "cold" or descriptive/inferential beliefs and "hot" or evaluative beliefs are interrelated, in CT they are often treated together as distorted 
cognitions and included as such in various distorted cognitions scales/measures (e.g., Automatic Thoughts Questionnaire-Short Version; Netemeyer et al. 2002). However, the distinction can have both theoretical (e.g., what type of cognitions is a proximal mediator of emotional distress) and practical (e.g., on what type of cognitions we should mainly focus our interventions) implications; therefore, its exploration is fundamental not only for the CBT field, but also for the clinical field in general. In a way, this kind of investigation is similar to that of phenomenology of perception versus its underlying cognitive mechanisms (i.e., stages), both being very important.

Another distinction regarding the cognition types targeted in CBT (in both CT and REBT) is the distinction between "core beliefs" (coded/represented as schemas), which are more general and not easily accessible (e.g., "I am a worthless person"), and more "specific beliefs" or "automatic thoughts", which are activated automatically in circumscribed situations (e.g., "I don't think I can go on"). While core beliefs are often descriptive and inferential beliefs in the form of dysfunctional attitudes in CT, in REBT, core beliefs are conceptualized as evaluations in the form of general rational and irrational beliefs. According to CBT theory (Beck 1995; Ellis 1994), core beliefs interact with various activating events to generate more specific beliefs in the form of automatic thoughts. With reference to core beliefs, the automatic thoughts refer to the specific values a given schema has in specific situations (David et al. 2004). Consequently, automatic thoughts are postulated to be proximal constructs to dysfunctional feelings and behaviors. Both core and specific beliefs (i.e., automatic thoughts) are assumed to be descriptive, inferential, or evaluative (David 2015).

Associations between each of these cognitive constructs and distress or psychopathology have been intensively investigated (e.g., David et al. 2002, 2005; DiLorenzo et al. 2007; Mogoase and Stefan 2013). However, only a few studies have examined the relationship between "hot" or evaluative beliefs and "cold" or descriptive/inferential beliefs with regard to their relative contributions to emotional distress (Cristea et al. 2013; Montgomery et al. 2007; Szentagotai and Freeman 2007; Vîsla et al. 2013). The results of these studies seem to challenge the postulates of REBT and appraisal theory of emotion and suggest that evaluative or irrational beliefs have an indirect effect on distress through descriptions/inferences. However, upon closer examination, the scale used to assess automatic contextual beliefs, i.e., the Automatic Thoughts Questionnaire-Short Version (ATQ-SV; Netemeyer et al. 2002), contains both descriptive/inferential beliefs and evaluative beliefs. Therefore, only explicitly differentiating between descriptive/inferential beliefs and evaluative beliefs and conducting separate analyses on these constructs will allow for more appropriate conclusions to be drawn regarding the relative contribution of the different types of situation-specific cognitions in the generation of emotions. The differential investigation of these cognition types will also allow the competing theories described above to be tested. Moreover, previous studies have measured descriptions/inferences as specific beliefs and evaluative or rational/ irrational beliefs as core beliefs; thus, not only that it is questionable that a more general belief can mediate a more specific belief, but practically it only confirms both the REBT and CT theories that more general beliefs, in interaction with 
different activating events, generate specific beliefs that further generate various psychological consequences (e.g., emotions, behaviors). Therefore, to test the theory, both descriptive/inferential beliefs and evaluative beliefs should be measured at the same level of generalizability.

\section{Objectives of the Study}

The aim of the present study was first, to delineate descriptive/inferential beliefs from evaluative beliefs within the ATQ-SV (Netemeyer et al. 2002). The second objective was to clarify the relative contributions of situation-specific descriptions/ inferences and evaluations to emotional distress. Although previous studies explored the relationship between beliefs and appraisal, to the best of our knowledge there are no studies that investigated this relationship in the context of distorted cognitions as targeted in CBT. Moreover, most of the time researchers and clinicians consider descriptions/inferences and evaluations interchangeable, and consequently combine them in a unitary score within related measures. However, although they may be mixed together at the phenomenological level and even load on the same factor (e.g., as anxiety and depression items do too), they are distinct cognitive mechanisms at psychological mechanistic level and thus, this distinction and its theoretical and clinical implications should be investigated empirically. The ideas presented in this section lead to the conclusion that, although descriptions and inferences may contribute to emotions, they may operate via evaluations/appraisal (i.e., rational and irrational beliefs). Therefore, we predict that descriptive/ inferential beliefs will have an indirect effect on emotional distress through evaluative beliefs.

\section{Method}

\section{Participants}

Seventy-four undergraduate students (mean age $=24.68, \mathrm{SD}=7.47$ ) took part in the study in exchange for course credit. The gender distribution was $18.9 \%$ males $(n=14)$ and $81.1 \%$ females $(n=60)$. All of the participants were recruited from classes offered by the psychology department of a Romanian university. Written informed consent was obtained from all participants prior to taking part in the study in accordance with university IRB guidelines.

\section{Measures}

Automatic thoughts were measured using the Automatic Thoughts QuestionnaireShort Version (ATQ-SV; Netemeyer et al. 2002). This instrument comprises 15 statements representing dysfunctional self-related automatic thoughts. The subject was instructed to rate these statements in terms of frequency of occurrence in their lives on a 5-point Likert scale, ranging from 1 (never) to 5 (almost always). Total scores range from 15 to 75 , with higher scores representing more dysfunctional 
automatic thoughts. The scale has shown satisfactory reliability and validity (Netemeyer et al. 2002). The ATQ-SV was adapted for the Romanian population (Moldovan 2007), showing excellent reliability (Cronbach's alpha $=.92$ ). Cronbach's alpha for this sample was .91. We also performed separate reliability analyses for the items that were further included in the category of descriptions/ inferences (Cronbach's alpha coefficient $=.81$ ) and for those included in the category of evaluations (Cronbach's alpha coefficient $=.86$ ). Total scores for descriptions/inferences category range from 8 to 40 , and for evaluations category from 7 to 35, with higher scores indicating more dysfunctional automatic thoughts in both categories.

Exam-related distress was assessed using the Profile of Mood States-Short Version (POMS-SV; DiLorenzo et al. 1999). This measure has 37 items and yields six subscale scores as well as a total mood disturbance score. The total score was used for these analyses. Respondents rated each item on a scale of 1 (not at all) to 4 (extremely). The total mean item score is derived, with higher scores indicating greater distress. The scale has shown satisfactory reliability and validity (DiLorenzo et al. 1999). Previous studies with Romanian-speaking samples demonstrated that POMS-SV could be successfully employed for the assessment of psychological distress (David et al. 2005). In the current sample, POMS-SV had a good internal consistency, with a Cronbach's alpha coefficient of .95 .

\section{Procedure}

We chose the period before a mandatory university exam as the specific situation studied because previous research (e.g., DiLorenzo et al. 2007, 2011) has demonstrated that this period is often perceived by an individual as very stressful and may negatively affect an individual's emotional health. In essence, the university exam serves as an ecological context for the study of distress and its cognitive sources. The exam was a multiple choice exam that asked students to recognize one or more correct answers among a set of options that included three or four wrong answers; moreover, a wrong answer canceled a good one. Therefore, it seemed highly plausible to assume that students taking this type of exam will experience a more intense level of distress compared to students taking an essay exam, for example.

On the day of the midterm exam, immediately prior to the exam (i.e., $30 \mathrm{~min}$ before), students completed measures of automatic thoughts and emotional distress after providing basic demographic information. Three trained CBT therapists (the first author, who was a doctoral student, and another two doctoral students from the first author's department) divided the ATQ-SV items into descriptions/inferences and evaluations by consensus (see "Appendix"). As part of their training in psychotherapy (required for accreditation in Romania), all therapists had received, among others, courses in cognitive-behavioral techniques, as well as cognitivebehavioral interventions for specific disorders. The raters discussed each item and only place it into one of the categories after all agreed. 


\section{Data Analysis}

Bivariate correlations and mediation analyses were performed. For the mediation analyses, we used the bootstrapping procedure for assessing indirect effects (Preacher and Hayes 2008) within the PROCESS (Hayes 2012) mediation script for SPSS. Given the inherent difficulties of estimating effect sizes for mediation procedures, the authors recommend a standardized index called kappa-squared (i.e., $\kappa^{2}$; Preacher and Kelley 2011), which represents the magnitude of the indirect effect relative to the maximum possible indirect effect, given the design of the study and the distributional particularities of the variables considered. Therefore, $\kappa^{2}$ was used as effect size measure for mediation models found to be significant, and the corresponding confidence intervals were computed. Preacher and Kelley (2011) state that no formal criteria exist for what values of $\kappa^{2}$ relate to small, medium, and large indirect effect sizes; however Cohen's guidelines (Cohen 1988, p. 79-81) of $.01, .09$, and .25 could be used as imperfect estimates for small, medium, and large effects, respectively.

\section{Results}

Descriptive Statistics and Correlations

Descriptive statistics for all variables are presented in Table 1. Correlations between all variables are also presented. Results suggest that the current sample experienced a high level of negative emotions $(\mathrm{M}=50.99, \mathrm{SD}=31.37)$, comparable with the scores obtained by DiLorenzo et al. (2007) on college students in similar real-life stressful situations (i.e., taking an exam or starting the semester). Correlations between all measured variables were positive, statistically significant, and ranged from moderate to strong.

Table 1 Descriptive statistics and correlations between descriptions/inferences, evaluations, and emotional distress

\begin{tabular}{|c|c|c|c|c|c|c|c|c|}
\hline & Min & Max & Med & M & SD & 1 & 2 & 3 \\
\hline \multicolumn{9}{|c|}{ Cognitive variables } \\
\hline $\begin{array}{c}\text { Descriptions/ } \\
\text { inferences }\end{array}$ & 8 & 32 & 13 & 14.47 & 5.02 & - & & \\
\hline Evaluations & 7 & 29 & 12 & 12.81 & 4.68 & .85 & - & \\
\hline \multicolumn{9}{|c|}{ Emotional variables } \\
\hline Distress & 1 & 131 & 43.5 & 50.99 & 31.37 & .68 & .70 & - \\
\hline
\end{tabular}

Min $=$ minimum, $M a x=$ maximum, $M e d=$ median, $M=$ mean, $S D=$ standard deviation; All correlations are statistically significant ( $p<.01$ Bonferroni Holm corrected for multiple comparisons) 


\section{Mediation Analyses}

For mediation analysis, we used bootstrapping tests with 5000 re-samples and reported a bias-corrected and accelerated confidence interval (Preacher and Hayes 2008). Mediation is considered to have taken place when the confidence interval for the estimation of the indirect effect does not contain 0 . We alternatively tested two possible mediation models: (1) a model in which evaluations mediate the relationship between descriptions/inferences and distress and (2) a model in which descriptions/inferences mediate the relationship between evaluations and distress. The results (see Fig. 1) favored the first model, in which the indirect effect of descriptions/inferences on examrelated distress through evaluations was significant, indirect effect $=2.37, \mathrm{SE}=.956$, $95 \%$ CI (bias-corrected and accelerated) $=.382$ to $4.141 ; \kappa^{2}=.273,95 \%$ CI (biascorrected) $=.058$ to .451 . Moreover, the direct effect of descriptions/inferences on distress was insignificant (direct effect $=1.881, \mathrm{SE}=.975,95 \% \mathrm{CI}$ [bias-corrected and accelerated] $=-.062$ to 3.836). The ratio of the indirect to total effect of descriptions/inferences to distress was $.557, \mathrm{SE}=.225,95 \% \mathrm{CI}$ (bias-corrected) $=$ .117 to 1.011 . Thus, approximately $56 \%$ of the total effect of descriptions/inferences on exam-related distress was explained by evaluations. There was no evidence of mediation in the alternative model (indirect effect $=1.71, \mathrm{SE}=.931,95 \% \mathrm{CI}$ [bias-corrected and accelerated] $=-.069$ to 3.578). Moreover, the direct effect of evaluations on distress in this second model was significant (direct effect $=2.99, \mathrm{SE}=1.22,95 \% \mathrm{CI}$ [bias-corrected and accelerated] $=.554$ to 5.426). A post hoc analysis of the sample size necessary for .8 power when using bias-corrected bootstrap test of mediation was done using the procedure recommended by Fritz and MacKinnon (2007). Therefore, based on the $\mathrm{a}$ and $\mathrm{b}$ path coefficients obtained, we would have needed a minimum of 34 participants to achieve a power of .8.

\section{Discussion}

The present study is the first to examine the associations between descriptive/ inferential automatic thoughts, evaluative automatic thoughts, and exam-related

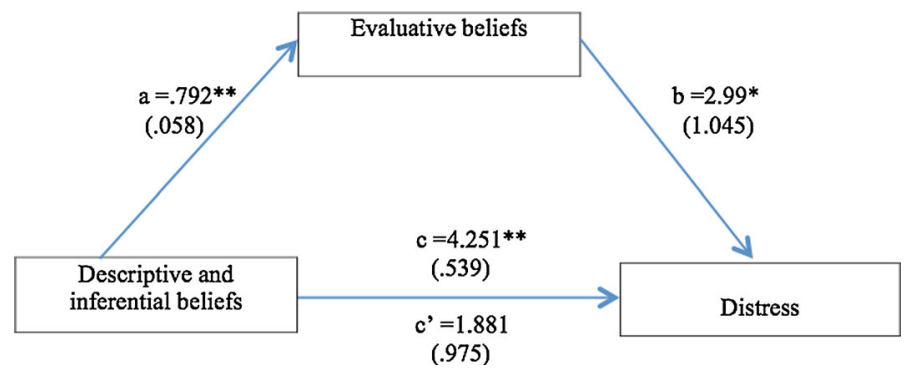

Fig. 1 Simple mediation diagram: $a, b, c$ and $c^{\prime}$ are path coefficients representing unstandardized regression weights and standard errors (in parentheses). The $c$ path coefficient represents the total effect of descriptive/inferential beliefs on exam-related distress. The $c$-prime path coefficient refers to the direct effect of descriptive/inferential beliefs on exam-related distress. All significant paths were marked with $* *$ if $p<.001$, and with * if $p<.05$ 
distress. In line with underlying theories (Ellis 1994; Lazarus 1991) as well as previous studies (e.g., David et al. 2002, 2005; DiLorenzo et al. 2007; Mogoase and Stefan 2013), the significant and medium-to-high positive associations between targeted cognitive constructs and exam-related distress confirmed our predictions. The significant and high positive correlation between descriptions/inferences and evaluations found in this study could be explained by both constructs being measured as situation-specific cognitions in the form of automatic thoughts. Similarly, Vîslă et al. (2013) obtained a higher correlation between different situation-specific cognitions (i.e., response expectancies and automatic thoughts) than between each of these situation-specific cognitions and core beliefs (i.e., general irrational beliefs). Both constructs were extracted from the same scale because there are currently no separate scales measuring these constructs. A selfreport scale was used for measuring the thought constructs, which could have introduced the shared method variance problem. Finally, both evaluative and descriptive/inferential beliefs were measured in the same context, a procedure that may have inflated the correlations.

Our meditational results are consistent with REBT theory (Ellis 1994) and appraisal theory of emotions (Lazarus 1991), in which descriptive/inferential beliefs affect distress via evaluative beliefs. The alternative model, the one in which descriptions and inferences mediate the relation between evaluations and emotional distress was not significant, meaning that descriptions and inferences are not mechanisms through which evaluations impact emotional distress. We used a robust method for testing mediation-bootstrapping — which has the advantage of being independent from sample sizes and not assuming a normal distribution of the indirect effects (Preacher and Hayes 2008). The values for our effect size (around .27) indicated that we managed to show a consistent part of the maximum indirect effect that could have been attained given the design and distribution characteristics. Based on Cohen's (1988) guidelines, we can conclude that our mediation model demonstrated a large effect size, with the confidence interval for the estimation of effect size containing .25 .

Moreover, the direct effect of descriptions/inferences on distress was insignificant, which is an indicator of total mediation. However, only approximately $56 \%$ of the total effect of descriptive/inferential beliefs on exam-related distress was explained by evaluations. Therefore, future studies are needed to investigate the role of other variables that may also have a mediating role on the relationship between descriptive/inferential beliefs and exam-related distress. One such variable could be unconscious information processing, which seems to be independent of beliefs or cognitive appraisal (LeDoux 2000). Moreover, in this study, the automatic thoughts questionnaire included only some of the irrational beliefs proposed by Ellis and Bernard (1985) as playing a central role in emotional distress and mental illnesses (e.g., demandingness/rigid thinking, frustration intolerance, and global evaluation). Notably, catastrophizing/awfulizing, the core irrational belief hypothesized to be involved in anxiety symptoms (Ellis 1994), was not measured. Also, the evaluative cognitions included in the ATQ-SV are not related to descriptive and inferential ones, in the sense that their topic is different. 
Based on the theoretical standpoint of classical CBT, these results reinforce the notion that in specific stressful situations, representations (i.e., descriptive and inferential beliefs) prime underlying cognitive vulnerability factors (i.e., evaluative beliefs or appraisals), which in turn are central components of the proximal mechanism resulting in emotional distress. Along the same lines, David et al. (2002) found that emotions are more directly associated with appraisal and irrational beliefs than with attributions. The current results also support the propositions of appraisal theory of emotions (Lazarus 1991). Despite the importance of representations as targets of personal evaluations with respect to their motivational relevance, the current results are compatible with the notion that representations may not produce emotions without first being appraised (Lazarus 1991).

From a clinical perspective, the results we obtained suggest that focusing on patients' descriptive and inferential beliefs (i.e., how they represent events in their lives) should be coupled with a focus on patients' evaluative cognitions (appraisal of events from their lives in terms of significance for their personal well-being; i.e., irrational beliefs). Clinicians that focus on restructuring descriptive and inferential cognitions only might not obtain a long lasting effect on emotional distress, but just a temporary one, in the sense of "feeling better" and not necessarily "getting better" (David et al. 2010). Moreover, most of the time descriptive and inferential cognitions are accurate (i.e., according to reality; e.g., "I failed the exam") and cannot be changed. Therefore, the intervention in this case will focus on changing the associated evaluative cognitions (i.e., "I must not fail the exam and if I do I'm worthless").

Extrapolating form automatic or specific cognitions as studied in this paper to core cognitions, some authors view schemas or core beliefs (especially evaluative), as more difficult to restructure than situation-specific thoughts (e.g., Beck 1995). Therefore, these authors suggest first changing automatic thoughts and then proceeding with core beliefs thereafter. Others argue (e.g., Ellis 1994) that the therapist should directly target core irrational beliefs, which can also lead to changes in automatic thoughts. However, automatic thoughts (mainly of the evaluative type) may occasionally be more difficult to modify than broader cognitions (e.g., it is more difficult to challenge the specific thought "I must be loved by my wife" than the more general thought "I must be loved by others") due to their strong emotional relevance. On the other hand, some people may argue that by changing the negative appraisal, we indirectly modify the probability that distorted descriptions and inferences will arise as well (Ellis 1994). However, experimental evidence for this hypothesis is mixed (see Bond and Dryden 2000), leaving space for future research to investigate it.

The present study has several limitations. The most important is its cross-sectional design, which does not allow any causal inferences to be made. However, the current results nonetheless provide some support for the observed associations between the tested constructs. Although we did measure both predictor and mediator prior to the outcome (distress), we did not measure actual change in either the mediator (evaluative beliefs) or the predictor (descriptive/inferential beliefs). A longitudinal experimental study comprising an intervention to modify the predictor variable and assessing the mediator before measuring change in outcome would strengthen arguments in favor of causal relationships. Secondly, our sample included 
significantly more women than men, not allowing for reliable gender comparisons. A more gender-balanced sample would be useful for testing whether there are gender differences in the relationship between cognitive constructs and exam-related distress. Third, to test our hypotheses, we extracted both the predictor and mediator from the same scale because there are currently no separate scales measuring these constructs. Therefore, future research would be well advised to invest in constructing new scales that separately assess both situation-specific appraisals and representations (i.e., descriptions and inferences). Forth, our sample of undergraduate students facing a mandatory university exam precludes generalization to other clinical and nonclinical samples in different stressful situations. Therefore, the results of the current study also need to be replicated in nonclinical samples as well as various clinical samples and in different stressful situations to establish the generalizability of the obtained model. Fifth, emotional distress was measured only from participant perspective; therefore, the association between automatic thoughts and outcome could vary upon who rates the emotional distress (i.e., participant, observer). Finally, it is possible that the delineation between descriptions/inferences and evaluations within the ATQ-SV to differ if we use another three experts in CBT or another procedure (e.g., independent categorization of cognitions and not by consensus). Therefore, future studies should replicate these results using independent categorization of the ATQ-SV items and reporting inter-rater reliability.

\section{Conclusion}

The study evaluated the relationship between descriptive/inferential and evaluative cognitions in regard to their relative contribution to emotional distress. The results suggest that descriptive and inferential cognitions have an indirect effect on distress through evaluative cognitions. However, descriptive/inferential cognitions do not independently (or directly) predict distress. Future studies could investigate the same relationships for core beliefs to determine which type of core beliefs or schema (evaluative or descriptive/inferential) is proximal to emotional distress. Finally, in a last step, future studies could use more complex statistical models, such as serial multiple mediator models (Hayes 2012), to test the relationship between evaluative and descriptive/inferential core beliefs (i.e., irrational beliefs and dysfunctional attitudes) and context-specific appraisals and descriptions/inferences (i.e., automatic thoughts) with regard to their relative contributions to emotional distress. These types of models would allow the mutual interplay of various potential mediators as well as their respective influences on distress to be investigated.

\section{Appendix}

Descriptions and inferences

2. I don't think I can go on.

4. Nothing feels good anymore.

6 . I can't get started. 
7. What's wrong with me?

9. I'll never make it.

13. My future is bleak.

14. It's just not worth it.

15. I can't finish anything.

\section{Evaluations}

1. I'm no good.

3. I'm so disappointed in myself.

5. I can't stand this anymore.

8. I'm worthless.

10. I feel so helpless.

11. Something has to change.

12. There must be something wrong with me.

\section{References}

Beck, A. T. (1976). Cognitive therapies and emotional disorders. New York: New American Library.

Beck, J. S. (1995). Cognitive therapy: Basics and beyond. New York: Guilford Press.

Bond, F. W., \& Dryden, W. (2000). How rational beliefs and irrational beliefs affect people's inferences: An experimental investigation. Behavioural and Cognitive Psychotherapy, 28, 33-43.

Broadbent, D. (1958). Perception and communication. London: Pergamon Press.

Chomsky, N. (1959). On certain formal properties of grammars. Information and Control, 2, 137-167. doi:10.1016/S0019-9958(59)90362-6.

Cohen, J. (1988). Statistical power analysis for the behavioral sciences (2nd ed.). Hillsdale, NJ: Lawrence Erlbaum Associates.

Cristea, I. A., Montgomery, G., Szamoskozi, S., \& David, D. (2013). Key constructs in "classical” and "new wave" cognitive-behavioral psychotherapies: Relationships among each other and with emotional distress. Journal of Clinical Psychology, 69, 584-599. doi:10.1002/jclp.21976.

David, D. (2015). Rational emotive behavior therapy. In R. L. Cautin \& S. O. Lilienfeld (Eds.), Encyclopedia of clinical psychology. Hoboken, NJ: Wiley-Blackwell.

David, D., Freeman, A., \& DiGiuseppe, R. (2010). Rational and irrational beliefs: Implications for mechanism of change and practice in psychotherapy. In D. David, S. J. Lynn, \& A. Ellis (Eds.), Rational and irrational beliefs: Research, theory, and clinical practice (pp. 195-217). New York: Oxford University Press.

David, D., Miclea, M., \& Opre, A. (2004). The information processing approach to the human mind: Basics and beyond. Journal of Clinical Psychology, 60, 353-368. doi:10.1002/jclp.10250.

David, D., Montgomery, G. H., Macavei, B., \& Bovbjerg, D. H. (2005). An empirical investigation of Albert Ellis's binary model of distress. Journal of Clinical Psychology, 61, 499-516. doi:10.1002/ jclp. 20058.

David, D., Schnur, J., \& Belloiu, A. (2002). Another search for the "hot" cognitions: Appraisal, irrational beliefs, attributions, and their relation to emotion. Journal of Rational-Emotive \& CognitiveBehavior Therapy, 20, 93-131. doi:10.1023/A:1019876601693.

David, D., \& Szentagotai, A. (2006). Cognitions in cognitive-behavioral psychotherapies; toward an integrative model. Clinical Psychology Review, 26, 284-298. doi:10.1016/j.cpr.2005.09.003.

DiLorenzo, T. A., Bovbjerg, D. H., Montgomery, G. H., Valdimarsdottir, H., \& Jacobsen, P. B. (1999). The application of a shortened version of the profile of mood states in a sample of breast cancer chemotherapy patients. British Journal of Health Psychology, 4, 315-325. doi:10.1348/ 135910799168669. 
DiLorenzo, T. A., David, D., \& Montgomery, G. H. (2007). The interrelations between irrational cognitive processes and distress in stressful academic settings. Personality and Individual Differences, 42, 765-776. doi:10.1007/s10942-006-0029-y.

DiLorenzo, T., David, D., \& Montgomery, G. H. (2011). The impact of general and specific rational and irrational beliefs on exam distress; a further investigation of the binary model of distress as an emotional regulation model. Journal of Cognitive \& Behavioral Psychotherapies, 11, 121-142.

Ellis, A. (1994). Reason and emotion in psychotherapy (Rev ed.). Secaucus, New Jersey: Birch Lane Press.

Ellis, A., \& Bernard, M. E. (1985). What is rational-emotive therapy (RET)? In A. Ellis \& M. E. Bernard (Eds.), Clinical applications of rational-emotive therapy (pp. 1-30). New York: Plenum Press.

Fritz, M. S., \& MacKinnon, D. P. (2007). Required sample size to detect the mediated effect. Psychological Science, 18, 233-239. doi:10.1111/j.1467-9280.2007.01882.x.

Hayes, A. F. (2012). PROCESS: A versatile computational tool for observed variable mediation, moderation, and conditional process modeling [white paper]. Retrieved from http://www.afhayes. com/public/process2012.pdf.

Hofmann, S. G., Asnaani, A., Vonk, I. J., Sawyer, A. T., \& Fang, A. (2012). The efficacy of cognitive behavioral therapy: A review of meta-analyses. Cognitive Therapy and Research, 36, 427-440. doi:10.1016/j.cpr.2005.07.003.

Lazarus, R. S. (1991). Emotion and adaptation. New York: Oxford University Press.

LeDoux, J. E. (2000). Emotion circuits in the brain. Annual review of neuroscience, 23, 155-184. doi:10. 1146/annurev.neuro.23.1.155.

Miller, G. A. (1956). The magical number seven, plus or minus two: Some limits on our capacity for processing information. Psychological Review, 63, 81-97. doi:10.1037/h0043158.

Mogoase, C., \& Stefan, S. (2013). Is there a difference between functional and dysfunctional negative emotions? The preliminary validation of the functional and dysfunctional negative emotions scale. Journal of Cognitive and Behavioral Psychotherapies, 13, 13-32.

Moldovan, R. (2007). Chestionarul gandurilor automate [Automatic thoughts questionnaire]. In D. David (Ed.), Sistem de evaluare clinica [Clinical Evaluation System]. Cluj-Napoca: RTS Publishing.

Montgomery, G. H., David, D., DiLorenzo, T. A., \& Schnur, J. B. (2007). Response expectancies and irrational beliefs predict exam-related distress. Journal of Rational-Emotive \& Cognitive-Behavior Therapy, 25, 17-34. doi:10.1007/s10942-006-0029-y.

Netemeyer, R. G., Williamson, D. A., Burton, S., Biswas, D., Jindal, S., Landreth, S., \& Primeaux, S. (2002). Psychometric properties of shortened versions of the automatic thoughts questionnaire. Educational and Psychological Measurement, 62, 111-129. doi:10.1177/0013164402062001008.

Preacher, K. J., \& Hayes, A. F. (2008). Asymptotic and resampling strategies for assessing and comparing indirect effects in multiple mediator models. Behavior Research Methods, 4, 879-891. doi:10.3758/ BRM.40.3.879.

Preacher, K. J., \& Kelley, K. (2011). Effect size measures for mediation models: Quantitative strategies for communicating indirect effects. Psychological Methods, 16, 93-115. doi:10.1037/a0022658.

Szentagotai, A., \& Freeman, A. (2007). An analysis of the relationship between irrational beliefs and automatic thoughts in predicting distress. Journal of Cognitive and Behavioral Psychotherapies, 7 , $1-9$.

Vîslă, A., Cristea, I. A., Szentágotai Tatar, A., \& David, D. (2013). Core beliefs, automatic thoughts and response expectancies in predicting public speaking anxiety. Personality and Individual Differences, 55, 856-859. doi:10.1016/j.paid.2013.06.003. 